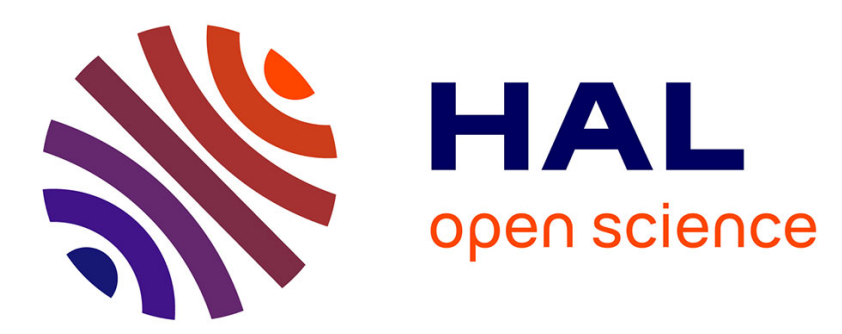

\title{
Pleine conscience dispositionnelle et comportements antisociaux : étude de profils chez le jeune adulte
}

Benoit Monié, Jonathan Bronchain, Sophie Becquié, Patrick Raynal, Henri Chabrol

\section{- To cite this version:}

Benoit Monié, Jonathan Bronchain, Sophie Becquié, Patrick Raynal, Henri Chabrol. Pleine conscience dispositionnelle et comportements antisociaux : étude de profils chez le jeune adulte. Journal de Thérapie Comportementale et Cognitive, 2019, 29 (1), pp.32-39. 10.1016/j.jtcc.2018.09.002 . hal03201640

\section{HAL Id: hal-03201640 \\ https://hal-univ-tlse2.archives-ouvertes.fr/hal-03201640}

Submitted on 19 Apr 2021

HAL is a multi-disciplinary open access archive for the deposit and dissemination of scientific research documents, whether they are published or not. The documents may come from teaching and research institutions in France or abroad, or from public or private research centers.
L'archive ouverte pluridisciplinaire HAL, est destinée au dépôt et à la diffusion de documents scientifiques de niveau recherche, publiés ou non, émanant des établissements d'enseignement et de recherche français ou étrangers, des laboratoires publics ou privés. 
Pleine conscience dispositionnelle et comportements antisociaux : étude de profils chez le jeune adulte

Dispositional mindfulness and antisocial behavior in young adult: A cluster-analytic study

Benoit Monié $^{\text {a }}$ Jonathan Bronchain ${ }^{\mathrm{a}}$, Sophie Becquiéa ${ }^{\mathrm{a}}$, Patrick Raynal ${ }^{\mathrm{a}}$, Henri Chabrol ${ }^{\mathrm{a}}$

a Centre d'Etudes et de Recherches en Psychopathologie et Psychologie de la Santé, Université Toulouse Jean jaurès, 5 Allée Antonio Machado, 31100 Toulouse, France

Auteur correspondant: Jonathan Bronchain, adresse électronique : jonathan.bronchain@etu.univ-tlse2.fr 


\section{Résumé}

Cette étude a pour but de comprendre les liens entre pleine conscience dispositionnelle, recherche de sensations et comportements antisociaux en utilisant des analyses de corrélations et classificatoires. Un échantillon de 1572 étudiants (âge moyen $=20,30 \pm 2,01$ ) a répondu à des questionnaires évaluant ces dimensions ainsi que la symptomatologie dépressive et les consommations d'alcool et de cannabis. Une analyse de classification basée sur les scores de pleine conscience et de recherche de sensations a permis d'obtenir quatre groupes très distincts : un groupe à bas traits (BT), un groupe avec des scores élevés de pleine conscience (HP), un troisième groupe avec un haut niveau de recherche de sensations (HS) et un dernier groupe avec des niveaux élevés de pleine conscience et de recherche de sensations (HPS). Le groupe HS présente des scores de comportements antisociaux significativement plus élevés que ceux des autres groupes, tandis que le groupe HP a un niveau de comportements antisociaux moins élevés que celui des trois autres groupes. Ces résultats suggèrent que des aptitudes à la pleine conscience pourraient modérer l'effet de la recherche de sensation élevée sur les comportements antisociaux. Cette étude offre des pistes pour la prévention et le traitement des comportements antisociaux à travers des interventions basées sur la pleine conscience.

Mots clés : Pleine conscience dispositionnelle; recherche de sensations ; comportements antisociaux ; psychopathologie ; étudiants. 


\begin{abstract}
Introduction. - Mindfulness skills and sensation seeking have been found to be differently associated with antisocial behavior in young adult. On the one hand, dispositional mindfulness is negatively associated with verbal aggression behaviors, anger, hostility and impulsivity (Brown \& Ryan, 2003; Heppner et al., 2008), and, on the other hand, it can reduce some aggressive behavior following a rejection situation (Heppner et al., 2008). In heightened mindfulness, situations generally perceived as threats are assessed differently and lead to more appropriate behaviors. Similarly, depressive symptomatology, cannabis use and alcohol use, seem to be related to dispositional mindfulness and antisocial behaviors (Karyadi et al., 2014; Royuela-Colomer \& Calvete, 2016). However, factors such as sensation seeking are important to take into account in the psychopathology of adolescents and young adults. Sensation seeking and dispositional mindfulness refer jointly to aspects of emotional regulation. Sensation seeking has been linked to the development of antisocial behaviors (Wilson \& Scarpa, 2011), and to proactive and reactive aggression in adolescents (PérezFuentes et al., 2016). Sensation seeking is also associated with alcohol use (LaBrie et al., 2014), and is an independent predictor of cannabis and alcohol use in adolescents (Chabrol et al., 2010). To our knowledge, no study has jointly highlighted sensation seeking and mindfulness to understand antisocial behavior. The aim of this study was to explore the links between dispositional mindfulness, sensation seeking and antisocial behavior using correlation and cluster analyzes.
\end{abstract}

Method. - Participants were 1,572 students from different French universities who completed self-report questionnaires assessing these dimensions as well as depressive symptomatology and alcohol and cannabis use.

Results. - Correlation analyzes show that dispositional mindfulness is negatively correlated with antisocial behavior $(r=-0.28, p<0.05)$ and with depressive symptoms $(r=-0.54, p$ 
$<0.05)$, while sensation seeking is positively correlated with alcohol $(r=0.26, p<0.05)$ and cannabis use $(r=0.27, p<0.05)$ and antisocial behavior $(r=0.18 ; p<0.05)$ (Table 1$)$. A classification analysis based on the dispositional mindfulness and sensation seeking scores yielded four distinct groups: a low-score cluster (BT), a high mindfulness cluster (HP), a third cluster with a high level of sensation seeking (HS) and a final cluster with high levels of mindfulness and sensation seeking (HPS) (Figure 1). The HS cluster, characterized by low levels of dispositional mindfulness and a high level of sensation seeking showed significantly higher scores of antisocial behavior, alcohol and cannabis use, and problematic cannabis use than those of the other groups (Table 2). The HS group also had a high level of depressive symptoms.

Discussion. - This result confirms the influence of sensation seeking on specific psychopathological factors (Chabrol et al., 2010; Pérez-Fuentes et al., 2016), particularly when dispositional mindfulness is low. The HP and HPS clusters, which are characterized by a high level of dispositional mindfulness, are distinguished by a significantly lower frequency of antisocial behavior and depressive symptoms. This result suggests that mindfulness may be a protective factor for antisocial behavior (Heppner et al., 2008) and depressive symptoms (Royuela-Colomer \& Calvete, 2016), regardless of the level of sensation seeking. There are limitations to this study. Firstly, the data collection was carried out through an online survey published on social networks. This mode of data collection can lead to an underrepresentation of some participant profiles, especially those with high impulsivity. Secondly, the use of self-report questionnaires can be problematic, although they have shown their validity in the assessment of dispositional mindfulness and antisocial behaviors (Bohlmeijer et al., 2011; Heeren et al., 2011). Thirdly, it is not known whether the results of the current study are applicable to a forensic sample, since all participants were university students. 
Conclusion. - Future research should include participants with forensic and high antisocial behavior. The results suggest paying particular attention to dispositional mindfulness in the understanding of specific psychopathological disorders. As behavioral disorders are regularly associated, mindfulness-based interventions, effective at different levels, could offer possibilities for the treatment of comorbid psychopathologies (Wupperman et al., 2015). Finally, mindfulness seems to be able to moderate the impact of certain factors such as sensation seeking.

Keywords: dispositional mindfulness; sensation seeking; antisocial behavior; college students; psychopathology. 


\section{Introduction}

Les dernières années ont vu se développer un nombre conséquent d'études sur la pleine conscience et ses bénéfices [1-3]. Sur le plan clinique, les interventions basées sur la pleine conscience ont montré une efficacité contre la symptomatologie dépressive, l'anxiété, les troubles du comportement alimentaire, les troubles liés aux substances psychoactives, et les traits de personnalité limite [4,5]. La pleine conscience est définie comme la capacité à diriger l'attention sur l'instant présent avec bienveillance et sans jugement, malgré la présence éventuelle d'événements psychologiques désagréables [6]. On appelle pleine conscience dispositionnelle l'aptitude naturelle, propre à chacun, à pratiquer la pleine conscience. Cette aptitude varierait d'un individu à l'autre et pourrait s'améliorer par la pratique [7,8]. La pleine conscience dispositionnelle a été envisagée de différentes façons. Ainsi, certains auteurs décrivent ce phénomène comme unidimensionnel [1,9-11], tandis que d'autres considèrent qu'il est l'expression de plusieurs composantes [12-16]. Ces différentes visions ont conduit au développement de plusieurs échelles d'évaluation, parmi lesquels le Five Facets Mindfulness Questionnaire (FFMQ) [16,17], un questionnaire auto-rapporté évaluant cinq facettes de la pleine conscience dispositionnelle : (1) l'observation, qui est la capacité à remarquer la présence d'expériences internes ou externes telles que des sensations, des cognitions, des émotions ; (2) la description, qui renvoie à la capacité à mettre des mots sur ses expériences internes ; (3) l'action en conscience, qui réfère à l'action en conscience et non-automatique ; (4) le non-jugement, qui est l'adoption d'une posture non évaluative envers ses propres pensées et sentiments ; (5) la non-réactivité, qui est la tendance à permettre à ses pensées et ses sentiments d'être libres d'aller et venir.

Les faibles dispositions à la pleine conscience se sont montrées liées à plusieurs indicateurs psychopathologiques parmi lesquels les comportements antisociaux auraient une place importante. Ainsi, la pleine conscience dispositionnelle auto-rapportée serait 
négativement associée aux comportements d'agression verbale, à la colère, l'hostilité, et l'impulsivité $[1,18]$. De même, l'induction d'un état de pleine conscience, par la pratique de la méditation, réduirait significativement les comportements d'agression suite à une situation de rejet [18]. La consommation de substances psychoactives serait également négativement associée à certaines facettes de la pleine conscience dispositionnelle telles que «action en conscience », «non-jugement » et «non-réactivité » $[19,20]$. Par conséquent, les interventions basées sur la pleine conscience réduiraient la consommation de plusieurs substances parmi lesquelles l'alcool, la cocaïne, les amphétamines, et le cannabis [21], démontrant leur pertinence dans la prise en charge des addictions [22]. Parallèlement, à l'exception d'«observation», toutes les facettes de la pleine conscience dispositionnelle seraient corrélées négativement à la symptomatologie dépressive [23]. De ce fait, les thérapies cognitives basées sur la pleine conscience ont démontré une efficacité dans la réduction de la symptomatologie des dépressions sévères [24].

D'autre part certains facteurs, comme la recherche de sensations, sont importants à prendre en considération dans la psychopathologie de l'adolescent et du jeune adulte. La recherche de sensations est un trait de personnalité défini par le besoin exigeant de rechercher des sensations et des expériences excitantes et intenses, et de s'engager dans des conduites de risques physiques et sociales, de rechercher le danger [25]. Par conséquent, les différentes échelles évaluant la recherche de sensations [26-28] ont montré des associations importantes avec l'implication dans des activités à risque et sports risqués, l'usage de drogues illégales et le trouble de la personnalité antisociale [29]. Le passage à l'acte antisocial peut consister en des actions incontrôlables, laissant peu de place à la volonté ou au consentement. Ainsi, la recherche de sensations a été reliée au développement de comportements antisociaux [30] et à l'agression proactive et réactive chez l'adolescent [31]. La recherche de sensations serait 
également associée à l'usage d'alcool [32] et serait un prédicteur indépendant de la consommation de cannabis et d'alcool chez l'adolescent [26].

Recherche de sensations et disposition à la pleine conscience renvoient conjointement à des aspects de la régulation émotionnelle. En effet, l'ouverture à l'expérience et la composante observation de la pleine conscience dispostionnelle seraient des caractéristiques communes à la fois aux conduites de recherche de sensations et aux pratiques de pleine conscience [33], si bien que certaines études ont retrouvé une association entre la recherche de sensations et la facette « observation » du FFMQ [34,35].

À notre connaissance, la littérature actuelle ne propose pas de mise en perspective de la recherche de sensations et des dispositions à la pleine conscience chez le jeune adulte. L'objectif de ce travail est donc d'étudier conjointement la recherche de sensations et la pleine conscience dispositionnelle dans leur lien avec les comportements antisociaux en utilisant des analyses classificatoires. L'usage de substances psychoactives, et la symptomatologie dépressive, régulièrement présents dans la psychopathologie du jeune adulte ont également été évalués. Cette approche, qui permet une prise en compte plus globale des individus, a pour but de révéler des profils spécifiques se différenciant sur ces variables psychopathologiques éventuelles cibles de traitements.

\section{Méthodologie}

\section{Participants et procédure}

Les données de cet échantillon ont été collectées par le biais d'un sondage en ligne qui a été diffusé auprès d'étudiants de différentes universités françaises. Ce sondage a été construit via le programme d'enquête Google Forms et le lien généré a été partagé sur les réseaux sociaux (Facebook) dans des groupes spécifiquement dédiés aux étudiants. L'échantillon final se composait de 1572 participants, 488 garçons (31\%) et 1084 filles (69\%) ; âge moyen garçons $=20.41 \pm 2.06$; âge moyen filles $=20.25 \pm 1.99$; rang $=(18-28)$. Le 
recueil de données s'est déroulé de décembre 2016 à février 2017. Concernant le domaine d'étude des participants, $31 \%$ d'entre eux étaient étudiants en sciences, ingénierie, $16 \%$ en économie, commerce, management et communication, $14 \%$ en sciences humaines, $13 \%$ en médical et paramédical, $8 \%$ en Lettres, $4 \%$ en éducation et pédagogie, $4 \%$ en Histoire, géographie, sciences politiques, $3 \%$ en droit, $2 \%$ en art et design, $1 \%$ en philosophie, $1 \%$ en Histoire de l'art et archéologie et 2\% étaient étudiants dans un autre domaine. Les objectifs de la recherche ont été présentés aux participants dès le début du questionnaire en précisant qu'il s'agissait d'une étude portant sur la personnalité et les comportements. Les participants ont été assurés de l'anonymat de leurs réponses.

\section{Mesures}

\section{Pleine conscience dispositionnelle}

Le Five Facets Mindfulness Questionnaire Short-Form (FFMQ-SF) [16,17] est composé de 24 items sur une échelle de Likert en cinq points évaluant les cinq facettes de la pleine conscience dispositionnelle : observation, description, action en conscience, nonréactivité, non-jugement (ex : «certaines de mes pensées me perturbent »).

\section{Comportements antisociaux}

Les comportements antisociaux ont été évalués à l'aide du Subtypes of Antisocial Behavior Questionnaire (STAB-Q) [36]. Ce questionnaire est composé de 32 items sur une échelle en cinq points évaluant trois sous-types de comportements : la violation de règles, l'agression sociale et l'agression physique (ex : « Se sentir mieux après avoir frappé »).

\section{Variables psychopathologiques émotionnelles}

La recherche de sensations a été évaluée avec la Brief Sensation Seeking Scale, BSSS [26,27]. Le BSSS est composé de huit items sur une échelle en cinq points. Un exemple d'item est : « J'aimerais faire du saut à l'élastique ». 
Le Patient Health Questionnaire-9, PHQ-9 [37,38] est composé de neuf items sur une échelle en quatre points permettant d'évaluer la symptomatologie dépressive. Un exemple d'item est : «peu d'intérêt ou de plaisir à faire les choses ».

Usage de cannabis et d'alcool

L'usage de cannabis a été mesuré à l'aide de la version française du Cannabis Use Disorders Identification Test-Revised (CUDIT-R) [39,40]. Il permet d'évaluer la consommation de cannabis au cours des six derniers mois ainsi que son utilisation problématique (ex : «Les jours où vous consommez du cannabis, vous vous sentiez défoncé(e) pendant combien d'heure(s) pour une journée type ?»).

La fréquence de consommation d'alcool au cours des trois derniers mois a été évaluée à l'aide de l'échelle de Likert en neuf points [41,42].

Analyses statistiques

Une analyse de classification a été utilisée pour identifier des groupes homogènes basés sur les scores standardisés de recherche de sensations et de pleine conscience dispositionnelle. Une analyse hiérarchique, utilisant la méthode d'agrégation de Ward et la distance euclidienne, a permis d'identifier le nombre de groupes en se basant sur le dendrogramme. La méthode des nuées dynamiques (K-means clustering) a ensuite été utilisée pour assigner chaque sujet à l'un des groupes identifiés. L'ensemble des analyses statistiques a été réalisé à l'aide du logiciel Statistica 10.

[mettre ici figure 1]

\section{Résultats}

\section{Statistiques descriptives et analyses corrélationnelles}

Les corrélations pour les différentes variables ont été reportées dans le Tableau 1.

Les analyses de corrélation montrent que la pleine conscience dispositionnelle est corrélée négativement avec les comportements antisociaux $(r=-0,28 ; p<0,05)$ et avec la 
symptomatologie dépressive $(r=-0,54 ; p<0,05)$, tandis que la recherche de sensations est corrélée positivement avec les consommations d'alcool $(r=0,26 ; p<0,05)$ et de cannabis $(\mathrm{r}$ $=0,27 ; p<0,05)$ et avec les comportements antisociaux $(r=0,18 ; p<0,05)$. Les principales statistiques descriptives sont présentées dans le Tableau 2.

\section{Analyse de classification}

L'analyse du coefficient d'agglomération et du dendrogramme a indiqué qu'une solution à quatre groupes était la plus appropriée. Une analyse discriminante a montré des discriminations nettes entre ces quatre groupes ( $\lambda$ de Wilks $=0,12 ; p<0,001)$ avec 96,3\% des participants correctement classés.

Le premier groupe $(n=287 ; 18 \%$ des participants), appelé « groupe bas traits » (BT) était caractérisé par un score de disposition à la pleine conscience peu élevé $(M=62,00 \pm$ $7,26)$ et un score de recherche de sensations peu élevé $(M=11,97 \pm 4,38$ ) (Figure 1 et Tableau 2). Le second groupe ( $n=405 ; 26 \%$ des participants) était caractérisé par un niveau élevé de disposition à la pleine conscience $(M=81,74 \pm 7,97)$ et un score de recherche de sensations faible $(M=12,74 \pm 3,90)$. Il a été nommé «groupe haute pleine conscience dispositionnelle » $(\mathrm{HP})$. Le troisième groupe $(n=465 ; 30 \%$ des participants), appelé «groupe haute recherche de sensations » (HS) était caractérisé par un haut niveau en recherche de sensations $(M=23,72 \pm 3,65)$ et un niveau peu élevé de disposition à la pleine conscience $(M=64,84 \pm 7,62)$. Le quatrième et dernier groupe $(n=414 ; 26 \%$ des participants), appelé «groupe haute pleine conscience et recherche de sensations (HPS) était caractérisé par un niveau élevé de pleine conscience dispositionnelle $(M=85,84 \pm 7,61)$ et un haut niveau en recherche de sensations $(M=22,68 \pm 3,78)$.

La comparaison des groupes sur les différentes variables, en utilisant des tests ANOVA et post-hoc, a montré que les groupes HP et HPS avaient significativement moins de comportements antisociaux et de symptômes dépressifs que les deux autres groupes (Tableau 
2). Le groupe HS avait des scores de comportements antisociaux, de consommations d'alcool et de cannabis, et d'usage problématique de cannabis significativement plus élevés que ceux des autres groupes, tandis que le groupe HP avait moins de symptômes dépressifs que les groupes BT et HS, des consommations d'alcool et de cannabis moins importantes que les groupes HS et HPS et un niveau de comportements antisociaux moins élevé que celui des trois autres groupes.

[mettre ici tableau 1 et 2]

\section{Discussion}

La littérature suggère que les faibles dispositions à la pleine conscience et la recherche de sensations seraient associées aux comportements antisociaux et à de nombreux indicateurs psychopathologiques. Cependant, à notre connaissance, il n'existe pas d'étude proposant une mise en perspective de ces deux variables par des analyses classificatoires. Concernant la validité de notre étude, nous pouvons souligner des niveaux de consistance interne satisfaisants pour l'ensemble de nos outils de mesure, avec des coefficients alpha de Cronbach situés entre 0,76 et 0,86 .

En accord avec la littérature, les résultats montrent des associations positives faibles mais significatives entre la recherche de sensations et les comportements antisociaux, l'usage de cannabis et la consommation d'alcool [31,32]. De même, la pleine conscience dispositionnelle auto-rapportée est corrélée négativement à la symptomatologie dépressive, et faiblement mais significativement corrélée aux comportements antisociaux [18,19,23].

Cette étude a retrouvé quatre groupes de sujets : «groupe bas traits » $(\mathrm{BT})$, «groupe haute pleine conscience dispositionnelle » $(\mathrm{HP})$, « groupe haute recherche de sensation » (HS), et «groupe haute pleine conscience et recherche de sensation » (HPS). Le groupe HS, caractérisé par de faibles dispositions à la pleine conscience et un niveau élevé de recherche de sensations, a montré des scores de comportements antisociaux, de consommation d'alcool 
et de cannabis, et d'usage problématique de cannabis significativement plus élevés que ceux des autres groupes. Le groupe HS présente également un niveau élevé de symptômes dépressifs. Ce résultat confirme l'implication de la recherche de sensation sur certains facteurs psychopathologiques [26,31], particulièrement lorsque les dispositions à la pleine conscience sont faibles. Les groupes HP et HPS, qui sont caractérisés par un niveau élevé de pleine conscience dispositionnelle, se distinguent par une fréquence significativement moins élevée de comportements antisociaux et de symptômes dépressifs. Ce résultat suggère que les dispositions à la pleine conscience pourraient être un facteur protecteur des comportements antisociaux [18] et de la symptomatologie dépressive [23], et ce indépendamment du niveau de recherche de sensations. En effet, pour le groupe HPS le niveau de pleine conscience dispositionnelle semble modérer les effets la recherche de sensation élevée. Enfin, le groupe HP se différencie des trois autres par des comportements antisociaux, des symptômes dépressifs et une consommation d'alcool peu élevés, suggérant que la pleine conscience dispositionnelle pourrait être un facteur de protection contre ces indices de désadaptation.

Cette étude présente certaines limites. Le recueil de données a été réalisé par le biais d'un sondage en ligne publié sur les réseaux sociaux. Ce mode de collecte de données, de par sa longueur, peut entrainer une sous-représentation de certains profils de participants, notamment ceux présentant une impulsivité importante. De plus, nous ne sommes pas en mesure d'affirmer que les résultats de l'étude actuelle sont applicables à un échantillon clinique, puisque tous les participants étaient des étudiants universitaires. Ainsi, les recherches futures devraient inclure des participants cliniques ou présentant des comportements antisociaux plus fréquents.

\section{Conclusion}

Nos résultats suggèrent de porter une attention particulière aux dispositions à la pleine conscience dans la compréhension de différents troubles psychopathologiques. Les troubles 
du comportement étant régulièrement associés entre eux, les interventions basées sur la pleine conscience, efficaces à différents niveaux, pourraient offrir des pistes pour le traitement des psychopathologies comorbides [43]. Enfin, les dispositions à la pleine conscience semblent pouvoir modérer l'impact de certains facteurs comme la recherche de sensations. Il serait donc intéressant de mener des études visant à déterminer les différents facteurs pouvant favoriser ou défavoriser de telles interventions.

\section{Déclaration de conflit d'intérêt}

Conflit d'intérêt : aucun. 


\section{Références}

[1] Brown KW, Ryan RM. The benefits of being present: mindfulness and its role in psychological well-being. J Pers Soc Psychol 2003;84(4):822-48.

[2] Davis DM, Hayes JA. What are the benefits of mindfulness? A practice review of psychotherapy-related research. Psychother 2011;48(2):198-208.

[3] Shapiro SL, Oman D, Thoresen CE, Plante TG, Flinders T. Cultivating mindfulness: Effects on well-being. J Clin Psychol 2008;64:840-62.

[4] Baer RA. Mindfulness training as a clinical intervention: a conceptual and empirical review. Clin Psychol Sci Pract 2003;10(2):125-43.

[5] Grossman P, Niemann L, Schmidt S, Walach H. Mindfulness-based stress reduction and health benefits: a metaanalysis. J Psychosom Res 2004;57(1):35-43.

[6] Kabat-Zinn J. Wherever you go, there you are: Mindfulness meditation in everyday life. New York: Hyperion. 1994

[7] Kuyken W, et al. How does mindfulness-based cognitive therapy work? Behav Res Ther 2010;48:1105-12.

[8] Papies EK, Barsalou LW, Custers R. Mindful attention prevents mindless impulses. Soc Psychol Pers Sci 2012;3:291-99.

[9] Bucheld N, Grossman P, Walach H. Measuring mindfulness in insight meditation (Vipassana) and meditation-based psychotherapy: the development of the Freiburg Mindfulness Inventory (FMI). J Meditation and Meditation Res 2001;1:11-34.

[10] Chadwick P, Hember M, Symes J, Peters E, Kuipers E, Dagnan D. (2008). Responding mindfully to unpleasant thoughts and images: reliability and validity of the Southampton Mindfulness Questionnaire (SMQ). Br J Clin Psychol 2008;47:45155. 
[11] Feldman G, Hayes A, Kumar S, Greeson J, Laurenceau JP. Mindfulness and emotion regulation: the development and initial validation of the Cognitive and Affective Mindfulness Scale-Revised (CAMS-R). J Psychopathol Behav Assess 2007;29:17790.

[12] Baer RA, Smith GT, Allen KB. Assessment of mindfulness by self-report: The Kentucky inventory of mindfulness skills. Assess 2004;11(3):191-206.

[13] Cardaciotto L, Herbert JD, Forman EM, Moitra E, Farrow V. The assessment of present-moment awareness and acceptance: The Philadelphia mindfulness scale. Assess 2008;2:204-23.

[14] Bodner TE, Langer EJ. Individual differences in mindfulness: the Mindfulness/Mindlessness Scale. Toronto: Poster presented at the 13th annual American Psychological Society Conference. 2001

[15] Bergomi C, Tschacher W, Kupper Z. Measuring mindfulness: first steps towards the development of a comprehensive mindfulness scale. Mindfulness 2013;4:18-32.

[16] Bohlmeijer E, ten Klooster PM, Fledderus M, Veehof M, Baer R. Psychometric properties of the five facet mindfulness questionnaire in depressed adults and development of a short form. Assess 2011;18(3):308-20.

[17] Heeren A, Douilliez C, Peschard V, Debrauwere L, Philippot P. Cross-cultural validity of the Five Facets Mindfulness Questionnaire: Adaptation and validation in a French speaking sample. Eur Rev Appl Psychol 2011;61(3):147-51

[18] Heppner WL, et al. Mindfulness as a means of reducing aggressive behavior: Dispositional and situational evidence. Agress Behav 2008;34:466-96.

[19] Karyadi KA, VanderVeen JD, Cyders MA. A meta-analysis of the relationship between trait mindfulness and substance use behaviors. Drug Alcohol Depend 2014;143:1-10. 
[20] Levin ME, Dalrymple K, Zimmerman M. Which facets of mindfulness predict the presence of substance use disorders in an outpatient psychiatric sample? Psychol Addict Behav 2014;28(2):498-506.

[21] Chiesa A, Serretti A. Are mindfulness-based interventions effective for substance use disorders? A systematic review of the evidence. Subst Use Misuse 2013;48:492-512.

[22] Johnson D, Mullen D, Smith ID, Wilson A. Mindfulness in addictions. BJPsych Adv 2016;22:412-19

[23] Royuela-Colomer E, Calvete E. Mindfulness facets and depression in adolescents: Rumination as a mediator. Mindfulness 2016;7:1092-1102.

[24] Eisendrath SJ, et al. A Randomized Controlled Trial of Mindfulness-Based Cognitive Therapy for Treatment-Resistant Depression. Psychother Psychosom 2016;85(2):99_ 110.

[25] Chabrol H. Traité de psychopathologie clinique et thérapeutique de l'adolescent. Paris: Dunod; 2011

[26] Chabrol H, Rodgers R, Sobolewski R, van Leeuwen N. Cannabis use and delinquent behaviors in a non-clinical sample of adolescents. Addict Behav 2010;35:263-65.

[27] Zuckerman M, Eysenck SBG, Eysenck HJ. Sensation seeking in England and America: cross cultural, age, and sex comparisons. J Consult Clin Psychol 1978;46:139-49.

[28] Zuckerman M. Zuckerman-Kuhlman Personality Questionnaire (ZKPQ): An alternative five-factorial model. (ed.) B. de Raad and M. Perugini. Big five assessment. Seattle: Hogrefe and Huber; 2002. p. 377-96.

[29] Zuckerman M. Sensation seeking and risky behavior. Washington, DC: American Psychological Association. 2007 
[30] Wilson LC, Scarpa A. The link between sensation seeking and aggression: A metaanalytic review. Aggress Behav 2011;37:81-90.

[31] Pérez-Fuentes MC, Molero MM, Carrión JJ, Mercader I, Gázquez JJ. Sensationseeking and impulsivity as predictors of reactive and proactive aggression in adolescents. Front Psychol 2016;7:1-8.

[32] LaBrie JW, Kenney SR, Napper LE, Miller K. Impulsivity and alcohol related risk among college students: Examining urgency, sensation seeking and the moderating influence of beliefs about alcohol's role in the college experience. Addict Behav 2014;39:159-64.

[33] Deplus S, Billieux J, Scharff C, Philippot P. A Mindfulness-Based Group Intervention for Enhancing Self-Regulation of Emotion in Late Childhood and Adolescence: A Pilot Study. Int J Ment Health Addict 2016;14(5):775-90.

[34] Murphy C, MacKillop J. Living in the here and now: interrelationships between impulsivity, mindfulness, and alcohol misuse. Psychopharmacol 2012;219:527-36.

[35] Peters JR, Erisman SM, Upton BT, Baer RA, Roemer L. A preliminary investigation of the relationships between dispositional mindfulness and impulsivity. Mindfulness 2011;2:228-35.

[36] Burt SA, Donnellan MB. Development and Validation of the Subtypes of Antisocial Behavior Questionnaire. Agress Behav 2009;35:376-98.

[37] Carballeira Y, Dumont P, Borgacci S. Criterion validity of the French version of Patient Health Questionnaire (PHQ) in a hospital department of internal medicine. Psychol Psychother 2007;80:69-77.

[38] Kroenke K, Spitzer RL,Williams JB. The PHQ-9: Validity of a brief depression severity measure. J Gen Intern Med 2001;16(9):606-13. 
[39] Adamson SJ, et al. An improved brief measure of cannabis misuse: the Cannabis Use Disorders Identification Test-Revised (CUDIT-R). Drug Alcohol Depend 2010;110:137-43.

[40] Chabrol H, Beck C, Laconi S. Contribution of health motive to cannabis high-school students. Addict Behav 2016;64:54-56.

[41] Simons J, Correia CJ, Carey KB, Borsari BE. Validating a five-factor motives measure: relations with use, problems and alcohol motives. J Couns Psychol 1998;45:265-73.

[42] Chauchard E, Goutaudier N, Valls M, Melioli T, van Leeuwen N, Chabrol H. Consommation de cannabis et comportements antisociaux dans un échantillon d'adolescents scolarisés. Encephale 2014;40:387-91.

[43] Wupperman P, Cohen MG, Haller DL, Flom P, Litt LC, Rounsaville BJ. Mindfulness and modification therapy for behavioral dysregulation: A comparison trial focused on substance use and aggression. J Clin Psychol 2015;71(10):964-78. 
Tableau 1 Corrélations entre les variables.

Correlations between variables.

\begin{tabular}{|c|c|c|c|c|c|c|}
\hline Variables & 1 & 2 & 3 & 4 & 5 & 6 \\
\hline 1. Pleine conscience dispositionnelle & - & & & & & \\
\hline 2. Recherche de sensations & $-0,02$ & - & & & & \\
\hline 3. Consommation de cannabis & $-0,04$ & $0,27 *$ & - & & & \\
\hline 4. Comportements antisociaux & $-0,28^{*}$ & $0,18^{*}$ & $0,17 *$ & - & & \\
\hline 5. Symptomatologie dépressive & $-0,54 *$ & 0,02 & $0,07 *$ & $0,25 *$ & - & \\
\hline 6. Consommation d'alcool & 0,01 & $0,26 *$ & $0,21 *$ & $0,10 *$ & $-0,03$ & - \\
\hline
\end{tabular}

$* p<0,05$. 
Tableau 2 Analyse de classification basée sur les scores de pleine conscience et de recherche de sensations. Comparaison des clusters par des tests ANOVA et post-hoc.

Classification analysis based on dispositional mindfulness and sensation seeking scores. Comparison of clusters by ANOVA and post-hoc tests.

\begin{tabular}{|c|c|c|c|c|c|c|c|c|c|c|}
\hline & $\begin{array}{l}\text { Echantillon } \\
N=1572\end{array}$ & $\begin{array}{l}\text { Min- } \\
\text { Max }\end{array}$ & Alpha & MIC & $\begin{array}{l}\text { Groupes } \\
M(E T)\end{array}$ & & & & & \\
\hline & & & & & BT & $\mathrm{HP}$ & HS & HPS & $F$ & $\begin{array}{l}\text { Comparaison } \\
\text { significatives }\end{array}$ \\
\hline & $M(E T)$ & & & & $n=287$ & $n=405$ & $n=465$ & $n=414$ & & \\
\hline $\begin{array}{l}\text { Pleine conscience } \\
\text { dispositionnelle }\end{array}$ & $73,81(11,60)$ & $19-117$ & 0,80 & 0,22 & $62,00(7,26)$ & $81,74(7,97)$ & $64,84(7,62)$ & $85,84(7,61)$ & $926,7 *$ & $\mathrm{HPS}>\mathrm{HP}>\mathrm{HS}>\mathrm{BT}$ \\
\hline $\begin{array}{l}\text { Recherche de } \\
\text { sensation }\end{array}$ & $18,47(6,64)$ & $0-32$ & 0,76 & 0,45 & $11,97(4,38)$ & $12,74(3,90)$ & $23,72(3,65)$ & $22,68(3,78)$ & $1005,07^{*}$ & $\mathrm{HS}>\mathrm{HPS}>\mathrm{HP}>\mathrm{BT}$ \\
\hline $\begin{array}{l}\text { Comportements } \\
\text { antisociaux }\end{array}$ & $24,12(13,03)$ & $0-124$ & 0,87 & 0,24 & $25,99(12,89)$ & $19,58(9,89)$ & $28,52(14,87)$ & $22,31(11,78)$ & $41,68^{*}$ & BT,HS >HP,HPS \\
\hline $\begin{array}{l}\text { Symptomatologie } \\
\text { dépressive }\end{array}$ & $11,66(5,54)$ & $0-27$ & 0,81 & 0,43 & $14,97(5,32)$ & $9,50(4,63)$ & $13,78(5,20)$ & $9,11(4,70)$ & $133,32 *$ & $\mathrm{BT}>\mathrm{HS}>\mathrm{HP}, \mathrm{HPS}$ \\
\hline $\begin{array}{l}\text { Consommation } \\
\text { d'alcool }\end{array}$ & $2,55(1,59)$ & $0-8$ & - & - & $2,07(0,58)$ & $2,24(1,55)$ & $2,92(1,58)$ & $2,78(1,54)$ & $25,98^{*}$ & HS,HPS >BT,HP \\
\hline $\begin{array}{l}\text { Consommation de } \\
\text { cannabis }\end{array}$ & $1,92(4,29)$ & $0-30$ & 0,86 & 0,61 & $0,79(2,48)$ & $0,82(2,58)$ & $3,32(5,89)$ & $2,21(4,00)$ & $34,75^{*}$ & HS $>$ HPS $>$ BT,HP \\
\hline
\end{tabular}

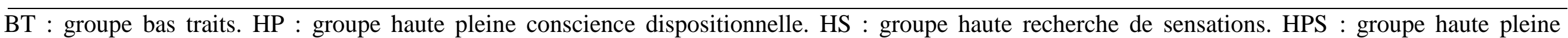
conscience et recherche de sensation. MIC: moyenne des corrélations inter-items. BT: low traits cluster. HP: high dispositional mindfulness cluster. HS: high sensation seeking cluster. HPS: high dispositional mindfulness and sensation seeking cluster. MIC: mean interitem correlation. * $\mathrm{p}<0,05$. 


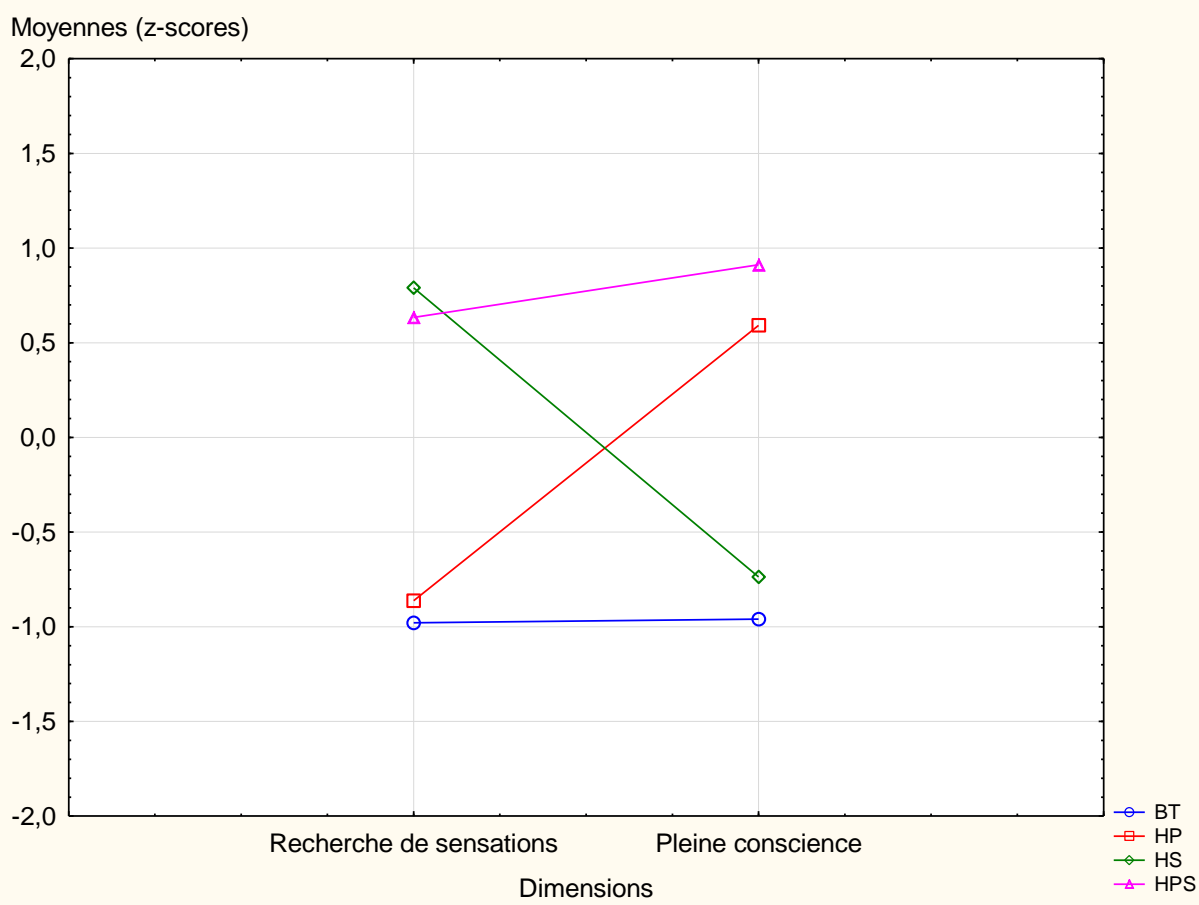

Figure 1 Classification en quatre groupes (BT, HP, HS et HPS) basée sur les scores aux dimensions de l'axe $x$. BT : Groupe bas traits. HP : groupe haute pleine conscience dispositionnelle. HS : groupe haute recherche de sensations. HPS : groupe haute pleine conscience et recherche de sensation.

Four-cluster solution (BT, HP, HS and HPS) based on scores for the FFMQ-SF and BSSS indicated on the $x$-axis. BT: low traits cluster. HP: high dispositional mindfulness cluster. HS: high sensation seeking cluster. HPS: high dispositional mindfulness and sensation seeking cluster. 